\title{
Ferromagnetic bond of Li10 cluster: An alternative approach in terms of effective ferromagnetic sites
}

Article in The Journal of Chemical Physics · September 2016

DOI: $10.1063 / 1.4961974$

CITATIONS

0

5 authors, including:

\section{Patricio Fuentealba}

University of Chile

156 PUBLICATIONS 4,783 CITATIONS

SEE PROFILE
READS

28

\section{Carlos A Cardenas}

University of Chile

38 PUBLICATIONS 393 CITATIONS

SEE PROFILE 


\section{AlP $\mid \begin{aligned} & \text { The Journal of } \\ & \text { Chemical Physics }\end{aligned}$}

Ferromagnetic bond of Li10 cluster: An alternative approach in terms of effective ferromagnetic sites

Roberto Donoso, Jaime Rössler, Sandra Llano-Gil, Patricio Fuentealba, and Carlos Cárdenas

Citation: The Journal of Chemical Physics 145, 094301 (2016); doi: 10.1063/1.4961974

View online: http://dx.doi.org/10.1063/1.4961974

View Table of Contents: http://scitation.aip.org/content/aip/journal/jcp/145/9?ver=pdfcov

Published by the AIP Publishing

\section{Articles you may be interested in}

The effect of sulfur covalent bonding on the electronic shells of silver clusters

J. Chem. Phys. 139, 164317 (2013); 10.1063/1.4827091

Strong spin-orbit effects in small Pt clusters: Geometric structure, magnetic isomers and anisotropy

J. Chem. Phys. 134, 034107 (2011); 10.1063/1.3530799

The role of quantum and thermal fluctuations upon properties of lithium clusters

J. Chem. Phys. 111, 5091 (1999); 10.1063/1.479765

Spin gap in low-dimensional Mott insulators with orbital degeneracy

J. Appl. Phys. 85, 5327 (1999); 10.1063/1.370241

Density functional study of structure and bonding in lithium clusters Li $\mathrm{n}$ and their oxides Li $\mathrm{n} O$

J. Chem. Phys. 106, 4566 (1997); 10.1063/1.473498

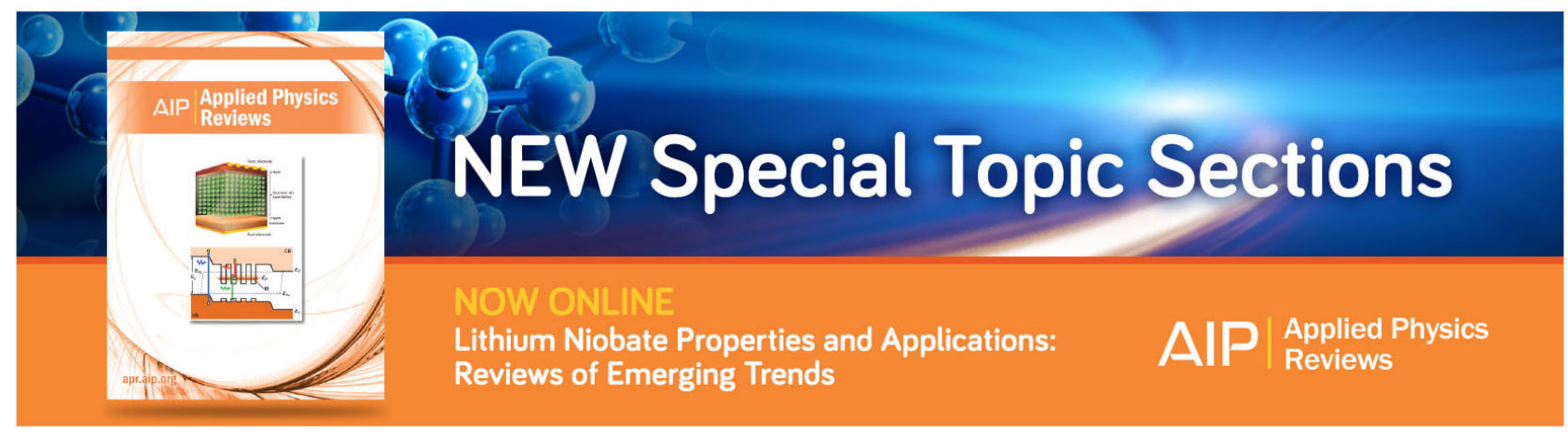




\title{
Ferromagnetic bond of $\mathrm{Li}_{10}$ cluster: An alternative approach in terms of effective ferromagnetic sites
}

\author{
Roberto Donoso, ${ }^{1,2}$ Jaime Rössler, ${ }^{1}$ Sandra Llano-Gil, ${ }^{3}$ Patricio Fuentealba, ${ }^{1,2, a)}$ \\ and Carlos Cárdenas ${ }^{1,2, a)}$ \\ ${ }^{1}$ Departamento de Física, Facultad de Ciencias, Universidad de Chile, Casilla 653, Santiago, Chile \\ ${ }^{2}$ Centro para el Desarrollo de la Nanociencia y la Nanotecnología (CEDENNA), Avda. Ecuador 3493, \\ Santiago 9170124, Chile \\ ${ }^{3}$ Faculty of Engineering, Food Engineering Program, Corporación Universitaria Lasallista, \\ Caldas, Antioquia, Colombia
}

(Received 6 June 2016; accepted 19 August 2016; published online 1 September 2016)

\begin{abstract}
In this work, a model to explain the unusual stability of atomic lithium clusters in their highest spin multiplicity is presented and used to describe the ferromagnetic bonding of high-spin $\mathrm{Li}_{10}$ and $\mathrm{Li}_{8}$ clusters. The model associates the (lack of-)fitness of Heisenberg Hamiltonian with the degree of (de-)localization of the valence electrons in the cluster. It is shown that a regular Heisenberg Hamiltonian with four coupling constants cannot fully explain the energy of the different spin states. However, a more simple model in which electrons are located not at the position of the nuclei but at the position of the attractors of the electron localization function succeeds in explaining the energy spectrum and, at the same time, explains the ferromagnetic bond found by Shaik using arguments of valence bond theory. In this way, two different points of view, one more often used in physics, the Heisenberg model, and the other in chemistry, valence bond, come to the same answer to explain those atypical bonds. Published by AIP Publishing. [http://dx.doi.org/10.1063/1.4961974]
\end{abstract}

\section{INTRODUCTION}

Chemical bond is the conceptual building-block of chemistry. All the ideas of stability, reactivity, and synthesis of molecular systems rely on the notion that atoms are held together by chemical bonds created by valence electrons that place between atoms, typically a pair of them. Although there is not a unique way to describe a bond in chemical physics because there is not an operator in quantum mechanics for it; the pairing of electrons of opposite spin is a signature of covalent, metallic, and ionic bonds. The traditional valence bond (VB) picture of a covalent bond is the result of the overlap of the atomic orbitals (AOs) of two (or more) atoms. This overlap accumulates electron density in the region between the atoms, which makes more attractive (negative) the nucleielectrons electrostatic interaction. But, this accumulation of density also increases the electron-electron repulsion. However, this increasing of electron-electron repulsion is less dramatic that one would expect from a classical behavior of electrons because of the Pauli principle (antisymmetry of the wavefunction) which keeps electrons of the same spin apart via the exchange "interaction." Then, the pairing of electrons of opposite spin helps to reduce the excess of kinetic energy due to the Pauli principle and so electrons in a bond form "localized" pairs of electrons of opposite spin. This gives physical support to Lewis structures where covalent bonds are depicted as paired electrons. Then, in a VB picture of the

\footnotetext{
a)Authors to whom correspondence should be addressed. Electronic addresses: pfuentea@ hotmail.es and cardena@macul.ciencias.uchile.cl
}

covalent bond, it is exchange and the pairing of electrons what makes the biggest contribution to the chemical bond. ${ }^{1}$

Imagine now a molecular system where all valence electrons are unpaired, that is, the system is in its state of maximum valence spin multiplicity. From the discussion above it seems counterintuitive, even paradoxical, that such system has chemical bonds. Yet, there is enough evidence that in some metals clusters, the state of maximum spin is bounded with respect to the free atoms. ${ }^{2-9}$ Further, these high-spin clusters are not mere theoretical entities but they have been observed in photo-physical experiments. ${ }^{10}$ Shaik et al. ${ }^{11,12}$ gave the name of ferromagnetic bonding to this type of bond in analogy, we believe, to the ferromagnetic ordering of local spins in ferromagnetic materials. A comprehensive and very clear discussion on the ferromagnetic bonding could be found in Refs. 12 and 13.

The simplest systems with ferromagnetic bonding are clusters of (n-)lithium atoms in its maximum spin multiplicity $(\mathrm{n}+1),{ }^{\mathrm{n}+1} \mathrm{Li}_{\mathrm{n}} .{ }^{8}$ Shaik et al. found that the binding energy per atom of these clusters becomes almost constant $(\approx 0.5 \mathrm{eV})$ for clusters with ten atoms and more. ${ }^{8,12}$ They explained the ferromagnetic bonding of $\mathrm{Li}$ clusters from the net binding curve of the ${ }^{3} \mathrm{Li}_{2}$ dimer. Using valence bond theory they showed that the mixing of $2 \mathrm{~s}$ and $2 \mathrm{p}$ AOs of $\mathrm{Li}$ produces excited covalent and ionic configurations that mix with the dominant repulsive covalent configuration in which each electron occupies a $2 \mathrm{~s}$ AO. This mixing with excited configurations (resonance) results in hybrids with reduced orbital overlap between same-spin electrons, which reduces the Pauli repulsion. This added to resonance-energy stabilization and delocalization is the source of stability of 
the net ferromagnetic interaction between $\mathrm{Li}$ atoms in the ${ }^{3} \mathrm{Li}_{2}$ dimer. With this understanding of the bond in ${ }^{3} \mathrm{Li}_{2}$, the authors also showed that that the stability of larger clusters could be rationalized as the sum of binding ferromagnetic interactions between pairs of nearest-neighbor atoms that allow valence electrons to be delocalized over the entire cluster. Their main conclusion is that the stability of high-spin Li clusters is attributable to the formation of delocalized ferromagnetic pairs. Finally, Alikhani and Shaik also used the electron localization function (ELF) to rationalize the ferromagnetic bonding in small Li clusters reaching a similar conclusion: bonds are multicenter with few delocalized electrons shared by more centers than the number of electrons in the bond. ${ }^{14}$ In this work, we focus on the clusters ${ }^{11} \mathrm{Li}_{10}$ and ${ }^{9} \mathrm{Li}_{8}$ to propose an alternative way to characterize the bonding in high-spin clusters. This new description uses Heisenberg exchange Hamiltonians, which are the simplest models to describe localized magnetism, to provide a neat picture of the (de-) localizability of the electrons and the stability of the clusters. This approach has the value of building a bridge between how bonding interactions in "magnetic" clusters are normally understood by physicists and chemists.

We focus in highly symmetric ${ }^{11} \mathrm{Li}_{10}$ because (i) for clusters with ten atoms and more the binding energy per $\mathrm{Li}$ atom becomes almost constant; therefore, we believe that $\mathrm{Li}_{10}$ is representative of larger clusters, and (ii) symmetry $\left(D_{4 d}\right)$ greatly reduces the dimension of the matrices of Heisenberg Hamiltonian to diagonalize as described in Section IV. However, we show that our model of effective ferromagnetic pairs holds in a less symmetric $\left(C_{2 v}\right)$ cluster like ${ }^{9} \mathrm{Li}_{8}$.

\section{COMPUTATIONAL METHODS}

All the geometries of the high-spin Lithium clusters were optimized at the unrestricted UB3LYP/6-311+G(d) level of theory. These structures were then symmetrized to the nearest point group allowing atoms to displace not more than $0.005 \AA$. Binding energies and all relative energies were evaluated from single point $\mathrm{CCSD} / 6-311+\mathrm{G}(\mathrm{d})$ calculations. All electronic structure calculations were done with Gaussian 09. ${ }^{15}$ The calculation of the electron localization function and its topological analysis were performed with the program TopMod of Silvi et al. ${ }^{16}$ The Heisenberg Hamiltonian of $\mathrm{Li}_{10}$ cluster was solved with the aid of Mathematica 10 to do the necessary linear algebra operations. Hartree-Fock exchange energy was printed in Gaussian 09 using the internal options (IOps) keywords and its value was verified with HORTON 2.0.0 $0^{17}$ which allows to print any contribution to the total energy. Atomic coordinates and energy of the optimized structures are given in the supplementary material.

\section{IMPORTANCE OF THE EXCHANGE IN THE STABILITY OF THE HIGH SPIN CLUSTER}

Shortly, a paramagnetic material is one in which the electron distribution and spin give rise to a net magnetic dipole moment that align with an external magnetic field. The main origin of this dipole is the spin of unpaired electrons whose spin is free to align with an external field. In ferromagnetic materials, the exchange interaction between those unpaired electrons is strong enough to overcome thermal motion and retain the alignment of the dipoles in the absence of an external field. Clearly, high-spin lithium clusters are not ferromagnetic in the standard sense because they are excited states and therefore cannot be permanent magnets. However, like in ferromagnetic materials, the exchange interaction between valence electrons plays a fundamental role in the bonding and stability of the high-spin cluster. To illustrate this we split the binding energy (with respect to free atoms) of ${ }^{11} \mathrm{Li}_{10}$ and ${ }^{1} \mathrm{Li}_{10}$ (at the geometry of ${ }^{11} \mathrm{Li}_{10}\left(@ D_{4 d}\right)$ ) in contributions from the exchange energy, $\Delta E_{e x}$, correlation energy, $\Delta E_{c o r r}$, classical repulsion energy (Hartree), $\Delta E_{H}$, nucleus-electron potential energy, $\Delta V_{N E}$, nucleus-nucleus potential energy, $\Delta V_{N N}$, and kinetic energy, $\Delta K$,

$$
\begin{aligned}
E_{b}\left[{ }^{11} \mathrm{Li}_{10}\right] & =E\left({ }^{11} \mathrm{Li}_{10}\right)-10 \times E\left({ }^{2} \mathrm{Li}\right) \\
& =\Delta E_{e x}+\Delta E_{\text {corr }}+\left(\Delta E_{H}+\Delta V_{N E}+\Delta V_{N N}\right)+\Delta K \\
& =-9.26-2.21+(7.17)-0.44=-4.75 \mathrm{eV}
\end{aligned}
$$

and

$$
\begin{aligned}
E_{b}\left[{ }^{1} \mathrm{Li}_{10}\right. & \left.\left(@ D_{4 d}\right)\right] \\
& =\Delta E_{e x}+\Delta E_{\text {corr }}+\left(\Delta E_{H}+\Delta V_{N E}+\Delta V_{N N}\right)+\Delta K \\
& =-3.31-5.69+(-2.59)+3.40=-8.20 \mathrm{eV},
\end{aligned}
$$

where correlation energy is evaluated at the CCSD/6-311+g(d) level of theory, while the rest of the contributions correspond to $H F / 6-311+g(d)$ values. The first to be noted is that in the low-spin cluster, exchange, correlation, and the nucleuselectron attraction contribute to the bonding, while in the high-spin cluster the main contribution comes from exchange followed by correlation. The main difference is that while in the low-spin cluster correlation makes the largest contribution, in the high-spin cluster the exchange is by far the most important contribution. The reduction of the kinetic energy also makes a small but meaningful contribution to the bonding of high-spin state. Then, the bonding in the low-spin cluster follows the typical picture of a covalent bond where there are attractive contributions from the classical potential energy $\left(\Delta E_{H}+\Delta V_{N E}+\Delta V_{N N}\right)$, exchange, and correlation, while the dominating contribution in the high-spin cluster is exchange. Therefore, a first conclusion raises attractive exchange interactions between unpaired electrons play a significant role in the stability of high-spin clusters, but it cannot be said that it is the only reason of stability. By un-pairing electrons, exchange energy becomes more dominant and correlation is reduced by a third because the average distance between pairs of electrons increases. This reduces the classical (Hartree) repulsion but also decreases the electron-nucleus attraction, which result in the net repulsive contribution of the classical potential energy to the high-spin cluster $(7.17 \mathrm{eV})$. However, there seems to be a key in the bonding of the high-spin cluster that the reduction of electron-nucleus attraction is limited, only $14 \mathrm{eV}$ less than in the low-spin case, and that the kinetic energy contributes to the bonding instead of the expected rise when higher energy MOs are occupied. The explanation to this lies in the nodal structure of the occupied MOs, which makes them all to have a "bonding character." This is easy to understand. Li has only one $2 \mathrm{~s}$ valence electron, so in a homoatomic 


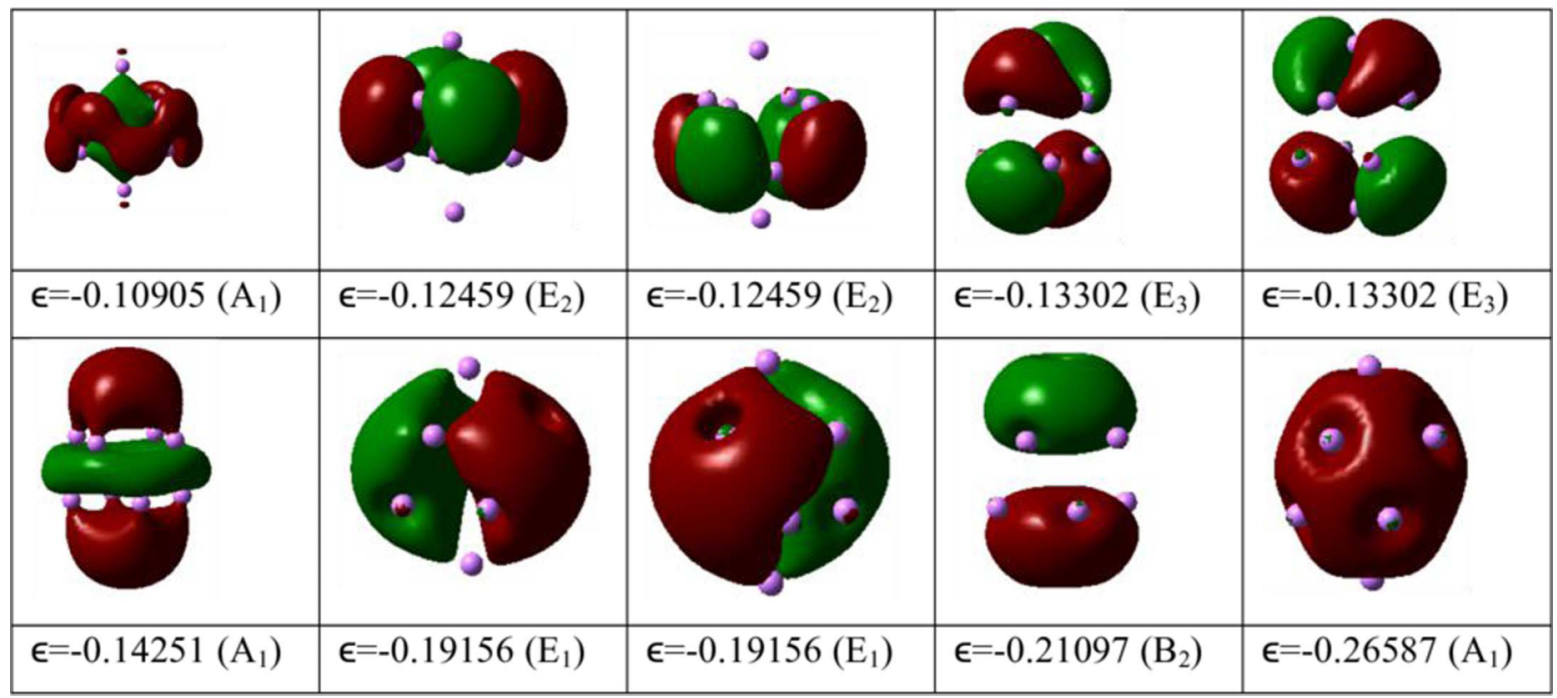

FIG. 1. Isosurfaces $\left(\psi= \pm 0.02 a_{0}^{-3}\right)$ of the ten valence molecular orbitals of the ${ }^{11} \mathrm{Li}_{10}$ cluster. Below every orbital it is indicated its B3LYP/6-311+G(d) Kohn-Sham eigenvalue (in $E_{h}$ ) and symmetry.

cluster with $\mathrm{N}$ atoms of $\mathrm{Li}$, the $2 \mathrm{~s}$ atomic orbitals combine to form $\mathrm{N}$ bonding orbitals and $\mathrm{N}$ antibonding orbitals. The homoatomic nature and the high symmetry make very likely that no antibonding MO lies lower in energy than the bonding ones. This, of course, is an oversimplified picture of the MO diagram because sp hybridization also occurs. Figure 1 shows isosurfaces of the ten valence occupied molecular orbitals of ${ }^{11} \mathrm{Li}_{10}$. It is clear that all of them have some bonding character because they have finite amplitude between subsets of atoms. This interpretation is in agreement with the link between the differences in the shape and the ordering of the MOs of high-spin $\mathrm{Li}$ and $\mathrm{Na}$ clusters and their bonding energy, as reported by Shaik et al. ${ }^{9}$ Hence, the ferromagnetic bond in the high-spin cluster ${ }^{11} \mathrm{Li}_{10}$ is possible because (i) a favorable attractive exchange "force" between electrons, (ii) a limited decreasing of the electron-nucleus attraction, and (iii) a null of even favorable decreasing of the kinetic energy. Note that the latter is consistent with the greater symmetry of the ${ }^{11} \mathrm{Li}_{10}$ $\left(D_{4 d}\right)$ compared to ${ }^{1} \mathrm{Li}_{10}\left(C_{4 v}\right)$.

\section{DELOCALIZABILITY OF BONDS}

The Heisenberg model of exchange interactions usually gives a very satisfactory explanation of the energy spectrum of magnetic materials provided that the electrons responsible for magnetism are localized around atoms in the valence bond (Heitler-London) sense. That is, the bond is mainly the result of the overlapping of atomic orbitals localized in the atomic positions with a small probability of hopping between sites. ${ }^{18}$ But, in cases where electrons responsible for magnetism are itinerant and delocalized over several atoms, the Heisenberg model fails to explain the energy spectrum and other properties. ${ }^{19}$ Hence, the Heisenberg model offers an appealing alternative to check how localized/delocalized the valence electrons of $\mathrm{Li}_{10}$ are. If electrons are localized in the VB sense, the Heisenberg model should satisfactorily explain the energy spectrum of the magnetic states with total spin $S=0,1,2,3,4$, and 5 .
In the Heisenberg model, exchange interactions are represented by the Hamiltonian

$$
\hat{\mathcal{H}}_{H}=-\sum_{j>i}^{n s i t e s} J_{i j} \vec{S}_{i} \cdot \vec{S}_{j},
$$

where $\vec{S}_{i}$ is the spin of the site(atom) $i$ and the coupling constants $J_{i j}$ measure the strength of the exchange interaction between spins in two different sites. If $J_{i j}>0$ a ferromagnetic (parallel spins) interaction is favored, while an antiferromagnetic interaction (pairing of spins) is favored if $J_{i j}<0$. The simplest Heisenberg model is the one in which only coupling between first neighbors is included; this coupling is the same for all pairs of atoms. However, this simplified model does not capture the correct symmetry of the nuclear framework $\left(D_{4 d}\right)$ of ${ }^{11} \mathrm{Li}_{10}$. In order to capture the correct symmetry and the three different distances between first neighbor atoms, a set of four different coupling constants is needed. ${ }^{20,21}$ Figure 2 schematically shows the structure of the Hamiltonian. If the cluster is depicted as two square-base pyramids opposed (and twisted $45^{\circ}$ ) by their faces, then the couplings that are considered to be different are the coupling between the vertex and the base of the pyramid $\left(J_{0}\right)$, the coupling between atoms in the pyramid $\left(J_{2}\right)$, the coupling between atoms of the bases of the pyramids $\left(J_{1}\right)$, and the coupling between a vertex and the distant base of a pyramid $\left(J_{3}\right)$. The Hilbert space of this Hamiltonian has $2^{10}=1024$ basis. To reduce the dimension of the matrices to diagonalize we have exploited the high symmetry of the molecular point group of this cluster $\left(D_{4 d}\right)$. The technical details can be found in the supplementary material. We made an exhaustive search in the space of coupling constants for those values that fit better the CCSD/6-311+g(d) energy of the states with spin multiplicity ranging from the singlet to the eleventh, that is, with states with spin multiplicity $\mathrm{M}=1,3,5,7,9$, and 11 (these states correspond, within the limitation of collinear-spin ab initio methods, to a total spin $S=0,1,2,3,4$, and 5). The parameters were forced to reproduce the difference of energy between the singlet and 


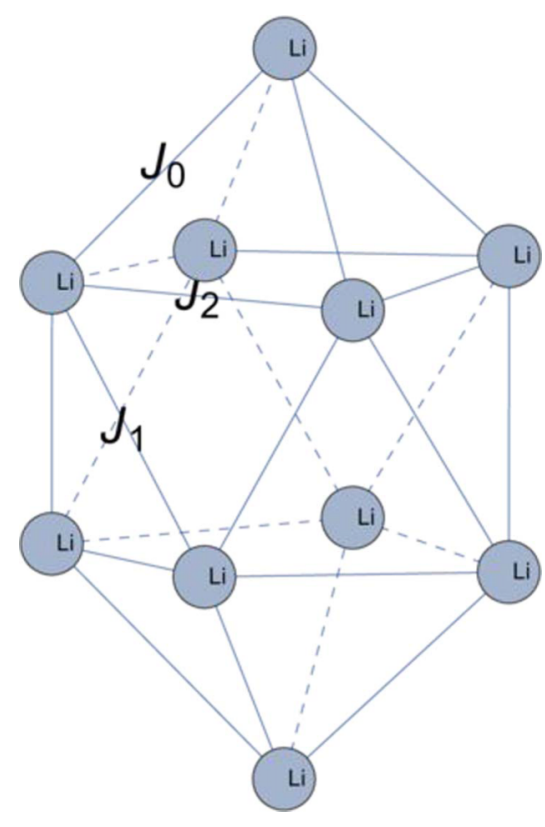

FIG. 2. Coupling constants of the ten-site Heisenberg Hamiltonian employed in Equation (3).

the highest spin states. The values of coupling constants were found to be $J_{0}=0.745 \mathrm{eV}, J_{1}=0.42 \mathrm{eV}, J_{2}=-0.20 \mathrm{eV}$, and $J_{3}=-0.36 \mathrm{eV}$. Note that the coupling between vertex and the base of the pyramid favors a ferromagnetic arrangement of the spins $(J>0)$. The same it is true for the coupling between atoms of both bases. The emergence of positive (ferromagnetic) coupling constants is a necessary condition to explain the structural stability of the cluster. All constants were positive and the cluster would not be structurally stable because a minimum energy solution will be attained with all atoms dissociated. As it can be seen from Table I, the spectrum of the Heisenberg model follows the trend of coupled-cluster singles-and-doubles (CCSD) calculations. If the Heisenberg Hamiltonian could perfectly fit the energy spectrum, valence electrons could be considered to be perfectly localized in (hybrid) orbitals at the atomic positions. ${ }^{22}$ Therefore, the deviation of the spectrum of the ${ }^{\mathrm{M}} \mathrm{Li}_{10}$ from the Heisenberg model indicates that valence electrons are not (perfectly) localized in the VB sense. This parallels the interpretation of Shaik et al. ${ }^{6,7,13}$ that delocalization of bonds and resonance play an important role in the stability of this type of clusters.
In Sec. V we show that a simple, but coherent, Heisenberglike model could be constructed in which valence electrons are depicted in terms of effective ferromagnetic sites. These sites are called effective because they do not necessary host an integer number of electrons but an effective number, and because they do not coincide with atomic positions. In the particular cases of ${ }^{11} \mathrm{Li}_{10}$ and ${ }^{9} \mathrm{Li}_{8}$, the valence electrons can be split into eight and four ferromagnetic pairs, respectively.

\section{A NEW PICTURE OF THE BOND IN TERMS OF EFFECTIVE FERROMAGNETIC CENTERS}

In a Heisenberg model of systems with no paired electrons it is reasonable to consider that the optimal sites for the model are those in which it is highly probable to find unpaired electrons. Those sites are the places where a "standing" electron will have a small chance to find a second electron of the same spin. The Taylor expansion of density of probability to find two electrons at an (spherical-) average distance $s$ around a point $\mathbf{r}$ given that the position of one of them is known with certainty is given by

$$
\begin{aligned}
& P(\mathbf{r}, s)=\frac{1}{3}\left(\sum_{i=1}^{o c c}\left|\nabla \psi_{i}(\mathbf{r})\right|^{2}-\frac{1}{4} \frac{|\nabla \rho(\mathbf{r})|^{2}}{\rho(\mathbf{r})}\right) s^{2}+\cdots, \\
& P(\mathbf{r}, s)=\frac{1}{3} D(\mathbf{r}) s^{2}+\cdots,
\end{aligned}
$$

where $\left\{\psi_{i}(\mathbf{r})\right\}$ is the occupied orbital and $\rho(\mathbf{r})$ is the electron density. Although Eq. (4) was originally deduced for a HartreeFock wavefunction, the interpretation of localizability that is given to the expression in parentheses, $D(\mathbf{r})$, remains valid within the Kohh-Sham method: the smaller the probability to find a second electron with the same spin, the more highly localized is the reference electron. Indeed, this is the key element of the electron localization function, ELF, of Becke and Edgecombe, ${ }^{23,24}$

$$
\eta(\mathbf{r})=\frac{1}{1+\left(\frac{D(\mathbf{r})}{D_{U E G}(\mathbf{r})}\right)^{2}},
$$

where $D_{U E G}(\mathbf{r})=3 / 5\left(6 \pi^{2}\right)^{2 / 3} \rho(\mathbf{r})^{5 / 3}$ is $D(\mathbf{r})$ for an noninteracting uniform electron gas. Therefore, a small probability $\frac{1}{3} D(\mathbf{r}) s^{2}$ corresponds to large values of the ELF. Figure 3 shows an isosurface of the ELF of ${ }^{11} \mathrm{Li}_{10}$ at $\eta(\mathbf{r})=0.43$. This value is very close to the largest value for which the regions

TABLE I. Relative energy (with respect to the singlet) of the six spin states of $\mathrm{Li}_{10}$ cluster. Fourth column corresponds to CCSD/6-311+G(d) calculations while fifth column corresponds to the best fit of a 10 -site and 10-electron Heisenberg Hamiltonian of Figure 2. As a measure of the spin contamination, the expected value of $\hat{\mathrm{S}}^{2}$ of the CCSD wave functions, before and after Löwdin annihilation, is also reported in the third column.

\begin{tabular}{lcccc}
\hline \hline Total spin (S) & $\begin{array}{c}\text { Spin multiplicity } \\
(2 \mathrm{~S}+1)\end{array}$ & $\begin{array}{c}\left\langle\mathrm{S}^{2}\right\rangle \text { Before/after } \\
\text { annihilation }\end{array}$ & $\begin{array}{c}\text { CCSD energy (eV) } \\
\text { (10-site, 10-electron) } \\
\text { Heisenberg model (eV) }\end{array}$ \\
\hline 0 & 1 & $0.0000 / 0.0000$ & 0.00 & 0.00 \\
1 & 3 & $2.2763 / 2.1566$ & 0.41 & 0.41 \\
2 & 5 & $6.5818 / 6.1014$ & 0.99 & 0.71 \\
4 & 7 & $12.2563 / 12.0089$ & 1.47 & 1.56 \\
5 & 9 & $20.1753 / 20.0000$ & 1.84 & 1.94 \\
\hline \hline
\end{tabular}




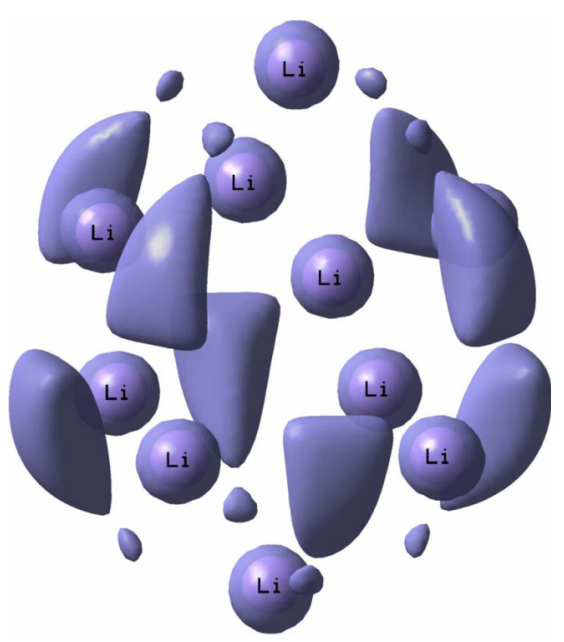

FIG. 3. Localization domains of ELF $=0.43$ of the ${ }^{11} \mathrm{Li}_{10}$ cluster. Within every lobe there is only one attractor of the gradient field of the ELF. Kohn-Sham orbitals UB3LYP/6-311+G(d) were used.

bounded by the ELF isosurfaces contain only one attractor (irreducible f-localization domains in the language of the topological analysis). The first to note is that, in this cluster, the ELF takes rather small values compared to systems with classic covalent bonds. For instance, in organic compounds analogous irreducible f-localization domains show up at $\eta(\mathbf{r}) \approx 0.75$. Although small values of ELF and a high variance (fluctuation) of the number of electrons within basins of ELF have been previously interpreted as a signature of the high delocalization of electrons in high spin Li clusters, ${ }^{14}$ the association of small values of ELF with delocalization has to be done with precaution for such small values of ELF. A simple argument is that the ELF of a homogeneous electron gas is 0.5 everywhere. Certainly, electrons in a homogeneous electron gas are more delocalized than electrons in a molecule or cluster because in the former all points of the space are equivalent. Then, the small values (less than 0.5) of ELF in ${ }^{11} \mathrm{Li}_{10}$ cannot only be associated to high delocalization but also to the fact that all valence electrons have the same spin. Besides, ELF values of open and closed shell systems are not completely comparable because there is not a unique way of incorporating the alpha and beta branches in Eq. (5). Nonetheless, the topology of the ELF is consistent with eight bonds located outside the faces of the polyhedral; each bond having 1.25 electrons spread in four centers.

More interesting, the partitioning of the space provided by the ELF gives a very appealing picture to construct a Heisenberg model where electrons are localized around the attractors of ELF (small $D(r)$ ), with each site hosting as many electrons as the integral of the electron density over the corresponding basin $\Omega$

$$
N_{\Omega}=\int_{\Omega} \rho(\mathbf{r}) d \mathbf{r},
$$

and a total spin given by (one half) the integral of the spin density over the basin

$$
S_{\Omega}=\frac{1}{2} \int_{\Omega}\left(\rho^{\alpha}(\mathbf{r})-\rho^{\beta}(\mathbf{r})\right) d \mathbf{r} .
$$

Black disks in Figure 4 show the position of the attractors of the ELF. There are in total 16 attractors that correspond to 8 pairs of equivalent basins. There is one of these pairs above every rhombohedra-like "face" of the cluster. The basin of the attractor labeled $\mathbf{A}$ holds around 0.82 electrons, while the basin of the attractor labeled $\mathbf{B}$ holds around 0.43 electrons. Similarly, the integrated spin density of basin $\mathbf{A}$ is 0.41 and 0.215 for basin $\mathbf{B}$. It should be noticed that the distance between attractors $\mathbf{A}$ and $\mathbf{B}(1.71 \AA)$ is considerably smaller

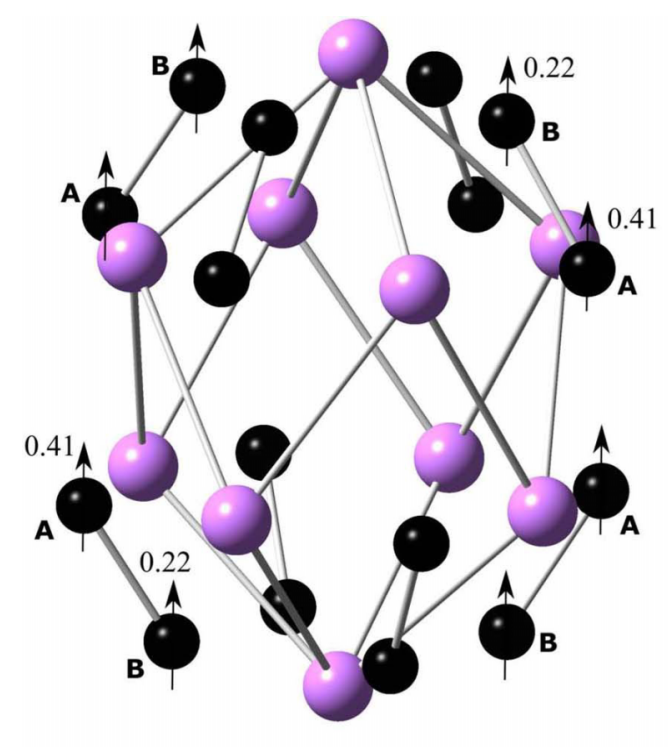

(a)

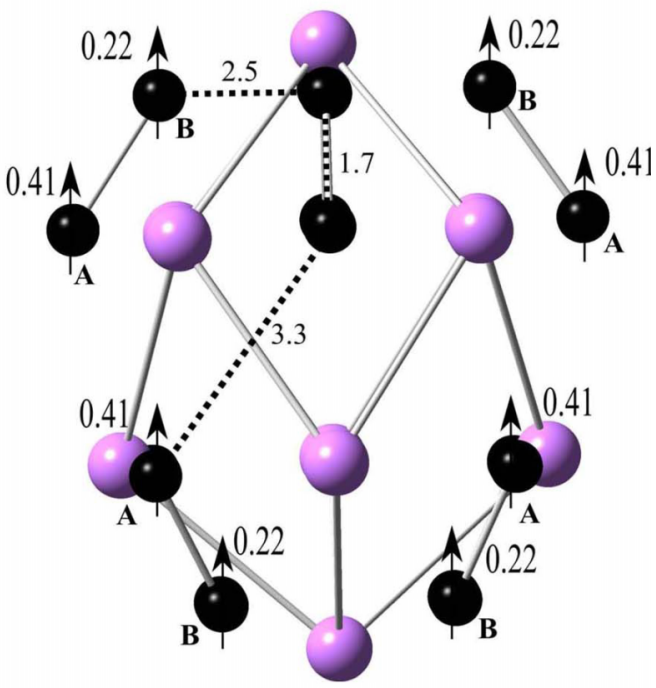

(b)

FIG. 4. Positions, in black, of the attractors of the ELF of the ${ }^{11} \mathrm{Li}_{10}$ cluster. Note that the 16 attractors form 8 equivalent pairs of attractors $\mathrm{A}$ and $\mathrm{B}$, which are interpreted (see text) as effective sites for a ferromagnetic bonding interaction of valence electrons. Numbers next to arrows correspond to the spin density integrated over each basin. The distance (in $\AA$ ) between attractors is also indicated. Panels (a) and (b) correspond to different views of the same cluster. All pairs are equivalent but some labels have been omitted for clarity of the figures. 


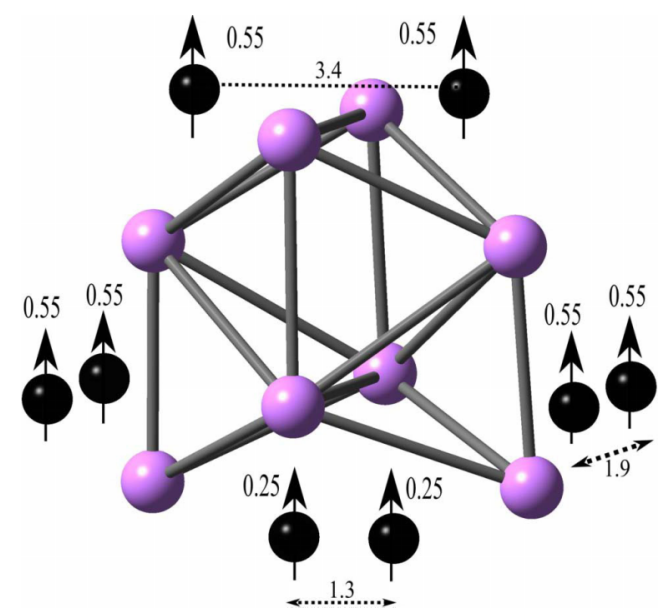

FIG. 5. Positions, in black, of the attractors of the ELF of the ${ }^{9} \mathrm{Li}_{8}$ cluster. Numbers next to arrows correspond to the spin density integrated over each basin. The distance (in $\AA$ ) between attractors is also indicated. Note how attractors come in pairs of effective sites for a ferromagnetic bonding interaction of valence electrons.

than the distances between next-neighbors attractors $(2.46 \AA$ and $3.31 \AA$ ). This provides insights to propose a model where electrons are localized around attractors $\mathbf{A}$ and $\mathbf{B}$ forming a "hetero-atomic-ferromagnetic" dimer with $\left|\vec{S}_{A}\right|=0.41$ and $\left|\vec{S}_{b}\right|=0.215$. In such a case, the exchange constant that couples electrons in $\mathbf{A}$ and $\mathbf{B}$ could be easily estimated from the energy of the system of highest spin, $E_{H S}$, and lowest spin, $E_{L S}$, which here were evaluated with the CCSD/6-311+G(d) method, ${ }^{25-27}$

$$
J=\frac{\frac{1}{8}\left(E_{L S}-E_{H S}\right)}{2 S_{A} S_{B}+S_{B}}=-1.104 \mathrm{eV} .
$$

To provide further evidence of our interpretation of the ferromagnetic bond in terms of effective ferromagnetic centers we have done the same analysis on the high-spin lithium cluster ${ }^{9} \mathrm{Li}_{8}\left(C_{2 v}\right)$. Figure 5 shows the position of the attractors of ELF and the integrated spin density of corresponding basins. It is clear that, likewise the ${ }^{11} \mathrm{Li}_{10}$ cluster, the attractors show a pairing structure with three different ferromagnetic dimers, which provide effective sites for a ferromagnetic bonding interaction of valence electrons. If a unique coupling constant is assumed for all of them, its value can be evaluated following similar reasoning to Equation (8): $J=-0.719 \mathrm{eV}$.

\section{SUMMARY}

We believe that a model where unpaired electrons establish effective ferromagnetic centers around the region of maximum probability is compatible with the observed electronic structures of ${ }^{11} \mathrm{Li}_{10}$ and ${ }^{9} \mathrm{Li}_{8}$. More interesting, this picture is compatible with the description of the bond provided by the electron localization function. In the case of the ELF the link is direct, electrons localized around the attractors of the ELF forming effective ferromagnetic sites whose spin is given by the integrated spin density. In the case of valence bond, the link is not that sharp but we adventure to postulate that these effective ferromagnetic sites are positions where optimal valence bond orbitals should be located. By optimal we mean that those orbitals will provide a localized (few resonances) picture of the valence electrons of the high-spin clusters ${ }^{11} \mathrm{Li}_{10}$ and ${ }^{9} \mathrm{Li}_{8}$. To further support our interpretation, isosurfaces of the electron density of ${ }^{11} \mathrm{Li}_{10}$ have been plotted in Figure 6. Note that the valence electrons accumulate just in the same regions of the effective ferromagnetic sites. Finally, we adventure that this model of effective ferromagnetic centers remains usable in more complex high-spin systems.

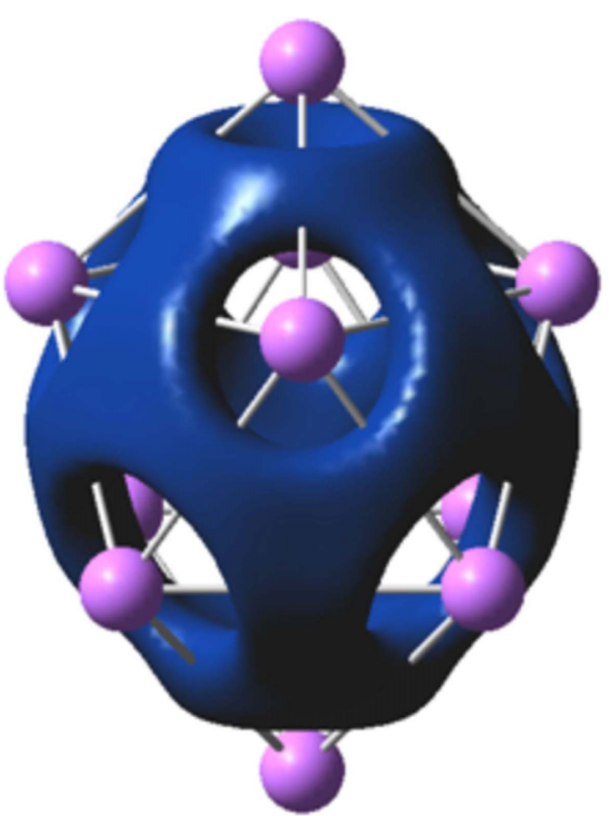

(a)

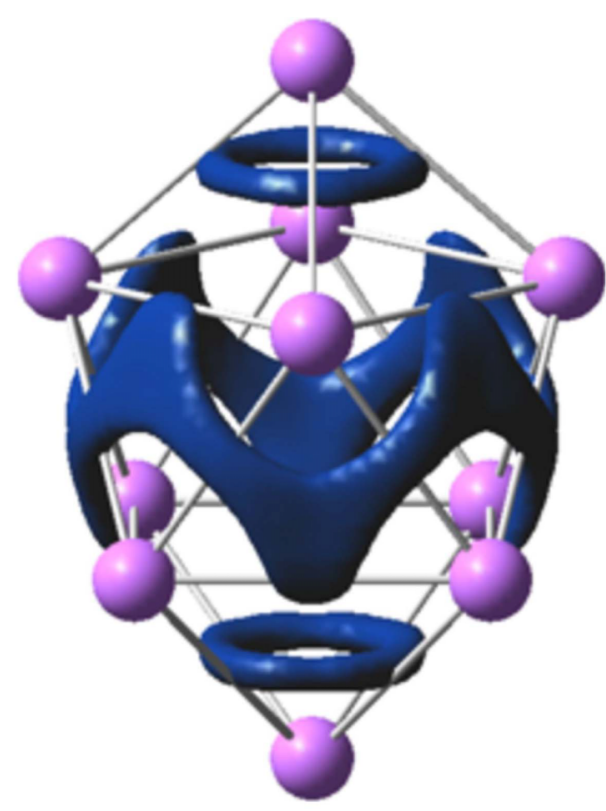

(b)

FIG. 6. Isosurfaces of valence electron density, computed with the UB3LYP/6-311+G(d), of the ${ }^{11} \mathrm{Li}_{10}$ cluster. (a) $\rho(\mathbf{r})=0.0075 a_{0}^{-3}$ and (b) $\rho$ (r) $=0.008 a_{0}^{-3}$. 


\section{SUPPLEMENTARY MATERIAL}

See supplementary material for atomic coordinates and energy of high-spin clusters and details on the diagonalization of the 10-electrons 10-sites Heisenberg Hamiltonian within the $\mathrm{D} 4 \mathrm{D}$ point group.

\section{ACKNOWLEDGMENTS}

This work was financed by FONDECYT through Project Nos. 1140313 and 1130202, and also by Centers Of Excellence With Basal/Conicyt Financing, Grant No. FB0807, CEDENNA. This paper is dedicated to Professor Alberto Vela on the occasion of his 65th birthday.

${ }^{1}$ G. Gallup, in Valence Bond Theory, edited by D. Cooper (Elsevier, 2002), Vol. 10.

${ }^{2}$ W. Kutzelnigg, V. Staemmler, and M. Gelus, Chem. Phys. Lett. 13(5), 496-500 (1972).

${ }^{3}$ M. L. Olson and D. D. Konowalow, Chem. Phys. 21(3), 393-399 (1977).

${ }^{4}$ M. N. Glukhovtsev and P. V. R. Schleyer, Isr. J. Chem. 33(4), 455-466 (1993).

${ }^{5}$ S. P. de Visser, Y. Alpert, D. Danovich, and S. Shaik, J. Phys. Chem. A 104(47), 11223-11231 (2000).

${ }^{6}$ D. Danovich and S. Shaik, J. Chem. Theory Comput. 6(5), 1479-1489 (2010).

${ }^{7}$ D. Danovich, W. Wu, and S. Shaik, J. Am. Chem. Soc. 121(13), 3165-3174 (1999).

${ }^{8}$ S. P. de Visser, D. Danovich, W. Wu, and S. Shaik, J. Phys. Chem. A 106(19), 4961-4969 (2002).

${ }^{9}$ S. P. de Visser, D. Danovich, and S. Shaik, Phys. Chem. Chem. Phys. 5(1), 158-164 (2003).

${ }^{10}$ J. Higgins, C. Callegari, J. Reho, F. Stienkemeier, W. E. Ernst, K. K. Lehmann, M. Gutowski, and G. Scoles, Science 273(5275), 629-631 (1996).

${ }^{11}$ S. P. de Visser, D. Kumar, M. Danovich, N. Nevo, D. Danovich, P. K. Sharma, W. Wu, and S. Shaik, J. Phys. Chem. A 110(27), 8510-8518 (2006).

${ }^{12}$ D. Danovich and S. Shaik, Acc. Chem. Res. 47(2), 417-426 (2013).

${ }^{13}$ D. Danovich and S. Shaik, Annu. Rev. Phys. Chem. 67(1), 419-439 (2016).

${ }^{14}$ M. Alikhani and S. Shaik, Theor. Chem. Acc. 116(4-5), 390-397 (2006).

${ }^{15}$ M. J. Frisch, G. W. Trucks, H. B. Schlegel, G. E. Scuseria, M. A. Robb, J. R. Cheeseman, G. Scalmani, V. Barone, B. Mennucci, G. A. Petersson,
H. Nakatsuji, M. Caricato, X. Li, H. P. Hratchian, A. F. Iszmaylov, J. Bloino, G. Zheng, J. L. Sonnenberg, M. Hada, M. Ehara, K. Toyota, R. Fukuda, J. Hasegawa, M. Ishida, T. Nakajima, Y. Honda, O. Kitao, H. Nakai, T. Vreven, J. A. Montgomery, Jr., J. E. Peralta, F. Ogliaro, M. Bearpark, J. J. Heyd, E. Brothers, K. N. Kudin, V. N. Staroverov, R. Kobayashi, J. Normand, K. Raghavachari, A. Rendell, J. C. Burant, S. S. Iyengar, J. Tomasi, M. Cossi, N. Rega, J. M. Millam, M. Klene, J. E. Knox, J. B. Cross, V. Bakken, C. Adamo, J. Jaramillo, R. Gomperts, R. E. Stratmann, O. Yazyev, A. J. Austin, R. Cammi, C. Pomelli, J. W. Ochterski, R. L. Martin, K. Morokuma, V. G. Zakrzewski, G. A. Voth, P. Salvador, J. J. Dannenberg, S. Dapprich, A. D. Daniels, O. Farkas, J. B. Foresman, J. V. Ortiz, J. Cioslowski, and D. J. Fox, GaUSSIAN 09, Revision A, Gaussian, Inc., Wallingford, CT, 2009.

${ }^{16}$ S. Noury, X. Krokidis, F. Fuster, and B. Silvi, Comput. Chem. 23(6), 597-604 (1999).

${ }^{17}$ T. Verstraelen, P. Tecmer, F. Heidar-Zadeh, K. Boguslawski, M. Chan, Y. Zhao, T. D. Kim, S. Vandenbrande, D. Yang, C. E. González-Espinoza, S. Fias, P. A. Limacher, D. Berrocal, A. Malek, and P. W. Ayers, HORTON 2.0.0, 2005, http://theochem.github.com/horton/.

${ }^{18} \mathrm{~K}$. Yosida, Theory of Magnetism (Springer Science \& Business Media, 1996).

${ }^{19}$ R. Stuart and W. Marshall, Phys. Rev. 120(2), 353-357 (1960).

${ }^{20}$ Despite its simplicity, the anti-ferromagnetic Heisenberg model captures essential ingredients in electron correlations. For instance, it can be shown that the anti-ferromagnetic Heisenberg model is a $t \ll U$ limit of the Hubbard model.

${ }^{21}$ K. A. Chao, J. Spalek, and A. M. Oles, Phys. Rev. B 18(7), 3453-3464 (1978).

${ }^{22}$ That the spectrum of Heisenberg Hamiltonian is not too far from the actual spectrum can be interpreted as the orbitals of the ${ }^{11} \mathrm{Li}_{10}$ cluster are neither pure Heitler-London nor Hund-Mulliken-Bloch orbitals, but a combination of both.

${ }^{23}$ A. D. Becke and K. E. Edgecombe, J. Chem. Phys. 92(9), 5397-5403 (1990).

${ }^{24}$ A. Savin, A. D. Becke, J. Flad, R. Nesper, H. Preuss, and H. G. Vonschnering, Angew. Chem., Int. Ed. Engl. 30(4), 409-412 (1991).

${ }^{25}$ L. Noodleman, J. Chem. Phys. 74(10), 5737-5743 (1981).

${ }^{26}$ E. Ruiz, J. Cano, S. Alvarez, and P. Alemany, J. Comput. Chem. 20(13), 1391-1400 (1999).

${ }^{27}$ This expression for the coupling constant of a dimer with two different local spins is in principle more appropriate when the energy of both states is evaluated with a single determinant method, and the energy of the singlet comes from a broken symmetry solution (unrestricted determinant). If one uses DFT (B3LYP/6-311+G(d)) with a broken solution for the singlet, the value of the constant does not change much, $\mathrm{J}=-1.42 \mathrm{eV}$. 